\title{
Endovascular Treatment Decisions in Patients with M2 Segment MCA Occlusions
}

\author{
(D) M. Almekhlafi, (D).M. Ospel, (D) G. Saposnik, (D) N. Kashani, (D) A. Demchuk, (D) M.D. Hill, (D) M. Goyal, and (D)B.K. Menon
}

\section{ABSTRACT}

BACKGROUND AND PURPOSE: Endovascular therapy in acute ischemic stroke is rapidly evolving. We explored physicians' treatment attitudes and practice in patients with acute ischemic stroke due to $\mathrm{M} 2$ occlusion, given the absence of Level-1 guidelines.

MATERIALS AND METHODS: We conducted an international multidisciplinary survey among physicians involved in acute stroke care. Respondents were presented with 10 of 22 case scenarios (4 with proximal M2 occlusions and 1 with a small-branch M2 occlusion) and asked about their treatment approach under A) current local resources, and B) assumed ideal conditions (no monetary or infrastructural restraints). Overall treatment decisions were evaluated; subgroup analyses by physician and patient baseline characteristics were performed.

RESULTS: A total of 607 physicians participated. Most of the respondents decided in favor of endovascular therapy in M2 occlusions, both under current local resources and assumed ideal conditions (65.4\% versus 69.6\%; $P=.017)$. Under current local resources, older patient age $(P<.001)$, longer time since symptom onset $(P<.001)$, high center endovascular therapy volume $(P<.001)$, high personal endovascular therapy volume $(P=.005)$, and neurosurgeons $(P<.001)$ were more likely to favor endovascular therapy. European respondents were less likely to favor endovascular therapy $(P=.001)$. Under assumed ideal conditions, older patient age $(P<.001)$, longer time since symptom onset $(P<.001)$, high center endovascular therapy volume $(P=.041)$, high personal endovascular therapy volume $(P=.002)$, and Asian respondents were more likely to favor endovascular therapy $(P=.037)$. Respondents with more experience $(P=.048)$ and high annual stroke thrombolysis treatment volume $(P=.001)$ were less likely to favor endovascular therapy.

CONCLUSIONS: Patients with M2 occlusions are considered appropriate candidates for endovascular therapy by most respondents in this survey, especially by those performing endovascular therapy more often and those practicing in high-volume centers.

ABBREVIATIONS: $\mathrm{EVT}$ = endovascular therapy; IQR = interquartile range

A cute ischemic stroke due to large arterial occlusions is a disabling condition with devastating consequences, unless timely reperfusion is achieved. ${ }^{1-3}$ Strokes caused by M2 segment MCA occlusions can present with severe symptoms and lead to large infarct volumes, with substantial morbidity and mortality. ${ }^{4}$ Recent endovascular stroke trials focused on patients likely to attain the greatest benefit from endovascular therapy (EVT). These trials mainly included patients with proximal (terminal

Received September 27, 2019; accepted after revision December 9.

From the Department of Clinical Neurosciences (M.A., J.M.O., A.D., M.D.H., M.G., B.K.M.), Hotchkiss Brain Institute; and Departments of Radiology (M.A., N.K., A.D., M.D.H., M.G., B.K.M.) and Community Health Sciences (M.A., M.D.H., B.K.M.), University of Calgary, Calgary, Alberta, Canada; Department of Radiology (I.M.O.), University Hospital Basel, University Basel, Basel, Switzerland; and Stroke Outcomes and Decision Neuroscience Research Unit (G.S.), Department of Medicine, and Li Ka Shing Knowledge Institute (G.S.), St. Michael's Hospital, University of Toronto, Toronto, Ontario, Canada

This study was supported by an unrestricted research grant from Stryker Inc. to the University of Calgary. The company was neither involved in the design and execution of the trial nor in the analysis and interpretation of its results. internal carotid "ICA" and proximal middle cerebral artery "M1") occlusions. ${ }^{5}$ Accordingly, current guidelines state that the benefit of EVT is uncertain in patients with M2 segment MCA occlusions; however, many operators believe that EVT is appropriate in these patients. ${ }^{6}$

Since the publication of the recent EVT trials ${ }^{5,7}$ in 2015, many studies have been published that report the safety and efficacy of EVT in patients with M2 segment MCA occlusions, including a patient-level meta-analysis from the Highly Effective Reperfusion evaluated in Multiple Endovascular Stroke trials Collaboration. ${ }^{4,7-11}$ Many operators now routinely perform EVT in patients with

Please address correspondence to Bijoy K. Menon, MD, Departments of Clinical Neurosciences, Radiology and Community Health Sciences, Foothills Medical Centre, University of Calgary, 1403 29th St. NW, Calgary, Alberta, T2N2T9, Canada; e-mail: docbijoymenon@gmail.com; @AlmekhlafiMa; @johanna_ospel; @NimaKashani; @bijoymenon; @mayank_G0

Indicates article with supplemental on-line table.

http://dx.doi.org/10.3174/ajnr.A6397 


\begin{tabular}{|c|c|c|c|}
\hline \multirow[b]{2}{*}{ Case Vignette } & \multicolumn{3}{|c|}{ EVT } \\
\hline & $\begin{array}{l}\text { Evidence } \\
\text { Level }\end{array}$ & $\begin{array}{l}\text { Rate under Current } \\
\text { Local Resources (\%) }\end{array}$ & $\begin{array}{l}\text { Rate under Assumed } \\
\text { Ideal Conditions (\%) }\end{array}$ \\
\hline $\begin{array}{l}\text { An 88-year-old, right-handed man has arrived at your hospital } \\
\text { at 10:00 AM with right hemiparesis and aphasia; symptom } \\
\text { onset was } 3 \text { hours ago; his stroke severity measured by } \\
\text { NIHSS is 14; ASPECTS on noncontrast CT is 7; baseline CTA } \\
\text { reveals a proximal left M2 segment MCA occlusion }\end{array}$ & $2 \mathrm{~B}$ & 81.6 & 85.9 \\
\hline $\begin{array}{l}\text { A } 45 \text {-year-old, left-handed man has arrived at your hospital at } \\
\text { 1:00 PM with left hemiparesis and visual field defect; he was } \\
\text { last seen as normal } 12 \text { hours ago; his stroke severity } \\
\text { measured by NIHSS is 15; ASPECTS on noncontrast CT is } 8 \text {; } \\
\text { baseline CTA shows a proximal right M2 segment MCA } \\
\text { occlusion }\end{array}$ & $2 B$ & 68.7 & 75.3 \\
\hline $\begin{array}{l}\text { A 94-year-old, left-handed woman has arrived at your hospital } \\
\text { at 2:00 AM with right hemiparesis and aphasia; symptom } \\
\text { onset was } 3.5 \text { hours ago; her stroke severity measured by } \\
\text { NIHSS is 12; ASPECTS on noncontrast CT is 7; the baseline } \\
\text { CTA shows a proximal left M2 segment MCA occlusion }\end{array}$ & $2 B$ & $60.3 \%$ & $67.2 \%$ \\
\hline $\begin{array}{l}\text { An } 85 \text {-year-old, right-handed woman has arrived at your } \\
\text { hospital at 11:00 PM with left hemiparesis; symptoms are } \\
3 \text { hours from onset; her stroke severity as measured by } \\
\text { NIHSS is 9; ASPECTS on noncontrast CT is 6; baseline CTA } \\
\text { shows a proximal right M2 segment MCA occlusion; she has } \\
\text { a history of mild cognitive impairment }\end{array}$ & $2 B$ & 62.3 & 63.4 \\
\hline $\begin{array}{l}\text { A 56-year-old, right-handed man has arrived at your hospital at } \\
\text { 3:00 PM with global aphasia; symptoms are } 3 \text { hours from } \\
\text { onset; his stroke severity measured by NIHSS is 8; ASPECTS } \\
\text { on noncontrast CT is 9; baseline CTA reveals a small-branch } \\
\text { left M2 segment MCA occlusion }\end{array}$ & $2 B$ & 53.8 & 56.4 \\
\hline
\end{tabular}

ischemic stroke and with M2 segment MCA occlusions. However, EVT decision-making in these patients is variable due to a lack of clear guideline-based treatment recommendations. Until now, little was known about how physicians make treatment decisions with regard to EVT in patients with acute ischemic stroke and with M2 segment MCA occlusions and what factors guide this process. By using a worldwide survey, this study aims to explore how physicians from different subspecialties manage patients with acute ischemic stroke due to M2 segment MCA occlusions.

\section{MATERIALS AND METHODS \\ Survey Design}

An international cross-sectional Web-based survey (UNMASKEVT) among stroke physicians and neurointerventionalists was conducted to understand the current treatment practice and decision-making with regard to using EVT in patients with acute ischemic stroke. ${ }^{12}$ Respondents were randomly assigned 10 of a pool of 22 ischemic stroke case scenarios (5 with ICA occlusion, 12 with M1 segment MCA occlusion, 5 with M2 segment MCA occlusion [4 proximal and 1 small-branch M2 segment MCA occlusion]). Respondents were then asked how they would treat the patient in the given scenario under local current resources and under assumed ideal conditions (ie, without monetary or infrastructural limitations). Response data were obtained from November 26, 2017, to March 27, 2018. Further details of the study have been published elsewhere (see the Table for all case vignettes). ${ }^{12}$

\section{Survey Respondents}

A total of 1330 stroke physicians (neurologists, interventional neuroradiologists, neurosurgeons, internists, geriatricians, and other physicians directly involved in acute stroke care) from 38 countries around the world were invited to participate in the survey by e-mail. No restrictions were applied with regard to years of experience or academic versus nonacademic background. Before accessing the case scenarios, the responding physicians provided personal and practice data, namely, age, sex, years of experience in stroke treatment, personal and center-specific estimated number of annual patients with stroke treated, geographic region, subspecialty, and hospital setting.

\section{Statistical Analysis}

Data are described by using standard descriptive statistics. Subgroup analyses used the $\chi^{2}$ test for differences in proportions and the nonparametric Wilcoxon rank sum test for differences in continuous data. Multivariable logistic regression with backward elimination was used to determine variables associated with an EVT decision among the 5 case scenarios with M2 segment MCA occlusions. All the models were adjusted for baseline ASPECTS, time from symptom onset to imaging, and patient age. Sensitivity analyses used mixed-effects logistic regression with "respondent" as a random-effects variable to account for hierarchical clustering of data within respondents. Two-sided $P<.05$ was considered statistically significant. All analyses were performed by using Stata version 15.1 (StatCorp, College Station, Texas).

\section{RESULTS}

Overall, 607 physicians (45.6\%) responded to the survey request. Across the 22 survey scenarios, $75.6 \%$ of the physicians favored 


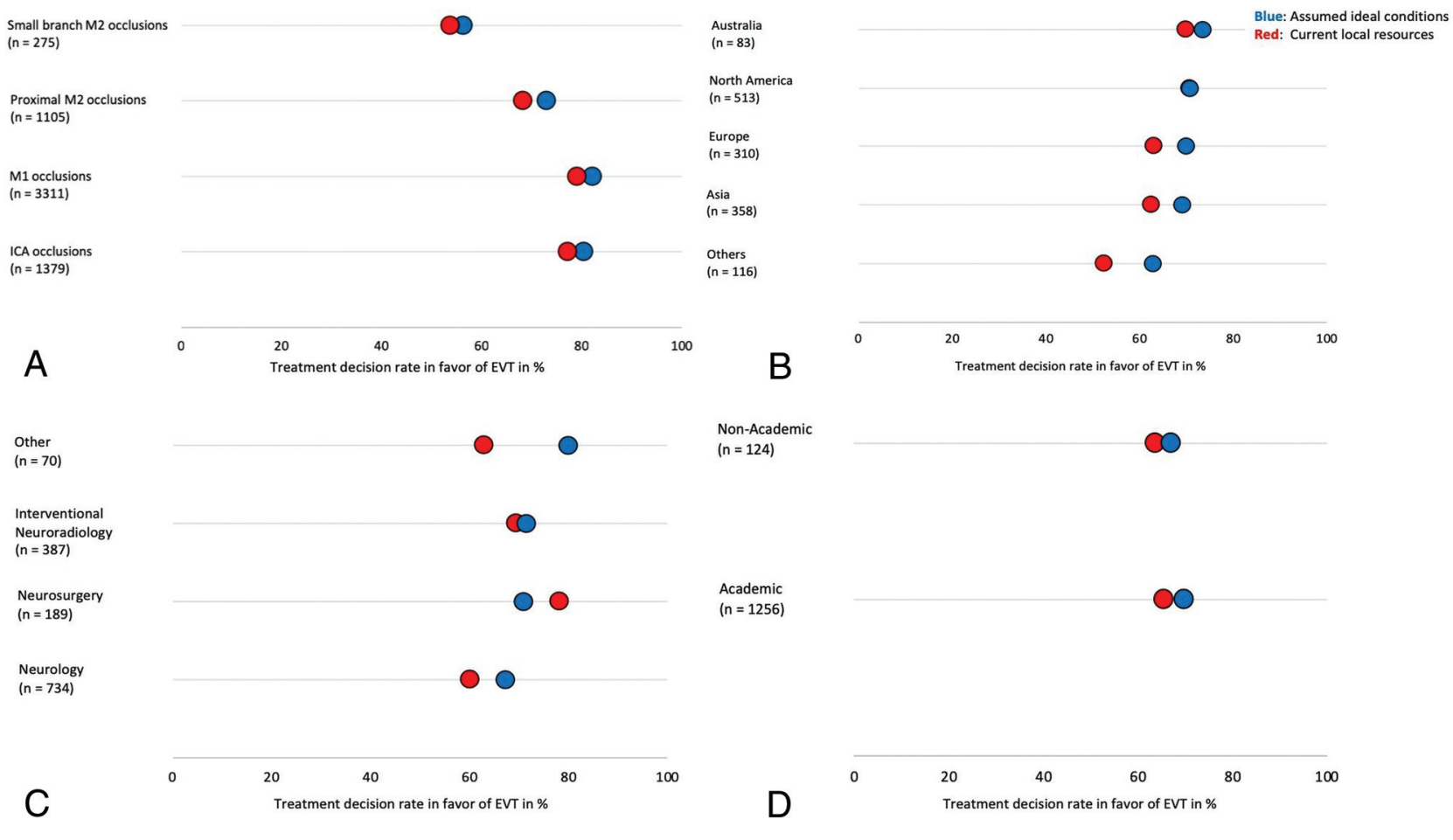

FIG 1. Decision rates in favor of EVT for different occlusion locations (A), decision rates in favor of EVT in M2 segment MCA occlusions for different geographic regions $(B)$, for different specialties $(C)$, and hospital settings $(D)$. Blue dots illustrate decision rates under assumed ideal conditions and red dots decision rates under current local resources. Decision rates under current resources and assuming ideal conditions in North America in $(B)$ were identical. Decision rates under current resources for neurosurgeons were lower than under assumed ideal conditions $(C)$.

using EVT (responses, 4586/6070) with current resources and 79.0\% under assumed ideal conditions (responses, 4793/6070). The EVT decision rate for M2 segment MCA occlusions was also high, given the current resources (65.4\%) and when assuming ideal conditions $(69.6 \%)(P=.017)$. Even in the scenario that describes a patient with a small-branch M2 segment MCA occlusion eligible for IV tPA, more than half of the respondents favored an EVT approach (53.8\% under current resources and $56.4 \%$ when assuming ideal conditions). However, these responses were lower than those for more proximal occlusions (ICA-M1 segment MCA, 78.6\% and $81.7 \%$ under current resources and assumed ideal conditions, respectively; $P<.001$ for difference in proportions) (Fig 1A). An overview of EVT decision rates under current resources and assumed ideal conditions for the 5 M2 segment MCA occlusion scenarios is provided in the Table. EVT decision rates under current resources and assumed ideal conditions across all countries for the $5 \mathrm{M} 2$ segment MCA occlusion scenarios are shown in Fig 2.

\section{Responses under Current Local Resources}

The EVT decision for M2 segment MCA occlusions differed significantly between regions under current local resources $(P<.001)$ (Fig 1B). Respondents from North America decided most often in favor of EVT $(70.8 \%)$, whereas those from "other regions" ranked lowest (52.6\%). The EVT decision also differed significantly across different specialties, with neurosurgeons ranking highest $(78.3 \%)$ and neurologists ranking lowest $(60.1 \%)(P<.001)$ (Fig 1C). The EVT decision between academic centers $(65.5 \%)$ and nonacademic centers $(63.7 \%)$ did not differ significantly $(P=.119)$ (Fig 1D).

Respondents who favored EVT for M2 segment MCA occlusions under current local resources performed significantly more endovascular procedures per year (median, 30 procedures; [interquartile range $\{\mathrm{IQR}\}, 30]$ versus 20 procedures [IQR, 30]; $P<$ .001) and worked in centers with a significantly higher annual EVT volume (median, 80 cases annually [IQR, 100] versus 40 [IQR, 70]; $P<.001$ ) and annual IV tPA volume (median, 100 cases annually [IQR, 125] versus 80 [IQR, 110]; $P<.001$ ) than those who refrained from using EVT. Survey respondents were younger (median age, 43 years [IQR, 12 years] versus 45 years [IQR, 13 years]; $P<.001$ ) and had fewer years of practice experience (median, 12 years [IQR, 13 years] versus 14.5 years [IQR, 13 years]; $P=.009$ ) than their peers who refrained from EVT.

\section{Multivariable Analyses}

In the multivariable analysis (On-line Table), the odds for choosing EVT for patients with M2 segment MCA occlusion were higher with increasing patient age (per decile increase in age, OR 1.02 [95\% CI, 1.01-1.02]; $P<.001$ ), time from symptom onset (per hour, OR 1.09 [95\% CI, 1.04-1.14]; $P<.001$ ), if respondents had a neurosurgical background (versus neurologists, OR 2.60 [95\% CI, 1.71-3.94]; $P<.001$ ), if the respondent's center had higher annual EVT case volume (per decile increase in case volume, OR 1.21 [95\% CI, 1.13-1.28]; $P<.001)$, if the respondents performed more EVT cases per year (per decile increase in case volume, OR 1.08 [95\% CI, 


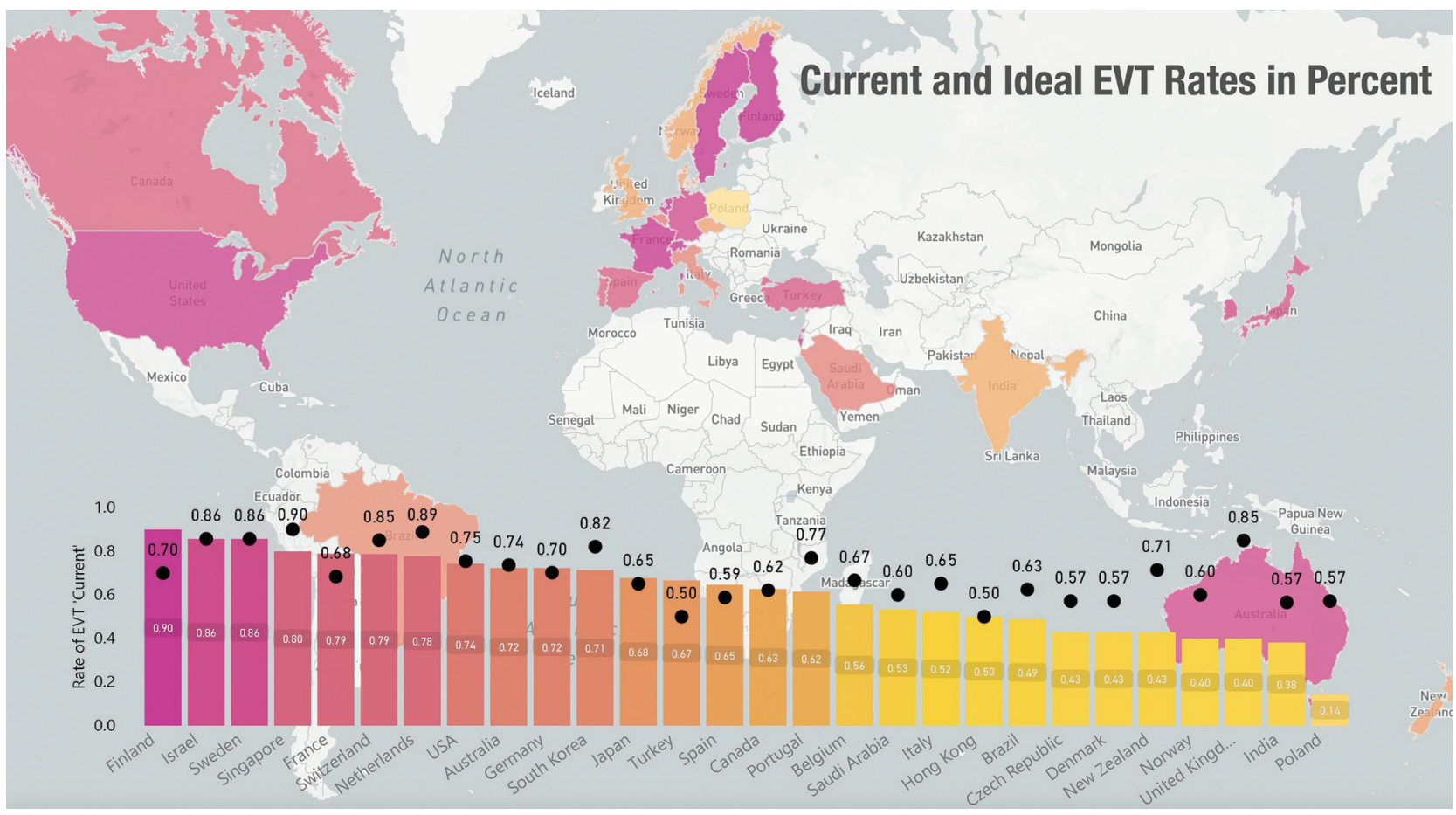

FIG 2. Decision rates in favor of EVT in patients with $M 2$ segment MCA occlusions by survey respondents across the globe, stratified by country, under current resources (bars), and assumed ideal conditions (black dots).

1.02-1.15]; $P=.005)$. Respondents from Europe were less likely to offer EVT (OR 0.68 [95\% CI, 0.49-0.94]; $P=.001$ ) when compared with the respondents from North America.

\section{Responses under Assumed Ideal Conditions}

The EVT decision for M2 segment MCA occlusions did not differ significantly between regions under assumed ideal conditions $(P=.486)$ (Fig $1 B)$. Respondents from Australia decided most often in favor of $\operatorname{EVT}(73.5 \%)$, whereas those from "other regions" ranked lowest (62.9\%). The EVT decision did not differ significantly across different specialties with "other specialties" ranking highest $(80.0 \%)$ and neurologists lowest (67.3\%) $(P=.099)$ (Fig 1C). The EVT decision between academic centers $(69.9 \%)$ and nonacademic centers $(66.9 \%)$ did not differ significantly $(P=.812)$ (Fig $1 D)$.

Respondents who favored using EVT for M2 segment MCA occlusions under assumed ideal conditions performed significantly more endovascular procedures per year (median, 30 [IQR, 35] versus 24 [IQR, 28]; $P<.001)$ and worked in centers with a significantly higher annual EVT volume (median, 75 cases annually [IQR, 97] versus 50 [IQR, 80]; $P<.001$ ) than those respondents who declined EVT. Survey respondents were younger (median age, 43 years [IQR, 11 years] versus 46 years [IQR, 13 years]; $P<.001$ ) and had fewer years of practice experience (median, 12 years [IQR, 11 years] versus 15 years [IQR, 13 years]; $P<.001)$ than their peers who refrained from using EVT.

\section{Multivariable Analyses}

In the multivariable analyses (On-line Table), the odds for choosing EVT were higher with increasing patient age (per decile increase in age, OR 1.02 [95\% CI, 1.01-1.03]; $P<.001$ ), time from symptom onset (per hour, OR 1.12 [95\% CI, 1.07-1.18]; $P<.001$ ), for respondents practicing in Asia (versus North America, OR 1.55 [95\% CI, 1.08-2.22]; $P=.037$ ), if the respondent's center had higher annual EVT case volume (per decile increase in case volume, OR 1.07 [95\% CI, 1.00-1.13]; $P=.041$ ), if the respondents performed more EVT cases per year (per decile increase in case volume, OR 1.09 [95\% CI, 1.03-1.16]; $P=.002$ ) were associated with higher odds of EVT use under assumed ideal conditions. The respondent's years of experience (per decile increase, OR 0.91 [95\% CI, 0.83-1.00]; $P=.048$ ) and personal annual stroke thrombolysis cases (per 10 case increase, OR 0.93 [95\% CI, 0.89-0.97]; $P=.001$ ) were associated with lower odds to proceed with EVT in patients with M2 segment MCA occlusion. The results were similar in sensitivity analyses when using mixed-effects modeling that accounted for clustering of response data within survey respondents (results not shown).

\section{DISCUSSION}

EVT decision-making for patients with an acute ischemic stroke and M2 segment MCA occlusions has been subject to debate because these patients were generally excluded from the largest EVT trials. In our study, most of the physicians who participated in this survey reported that they favored EVT in patients with acute ischemic stroke with M2 segment MCA occlusion. This reflects confidence in EVT as a safe and effective treatment option, even beyond large-vessel occlusions. The small but significant gap between current and ideal treatment decisions indicates that, although the respondents believed that EVT should be performed, their environment did not yet allow them to do so. This might change in the future as more evidence for safety and 
efficacy of EVT in M2 segment MCA occlusions becomes available. ${ }^{4,7-11}$

Survey respondents were more likely to predict that they would treat patients with ICA-M1 segment MCA occlusions with EVT than patients with M2 segment MCA occlusions. This most likely reflects current guidelines, which assign Class-1 Level-A evidence for EVT only in proximal (ICA-M1 segment MCA) occlusions. ${ }^{6}$ Analyses of the influence of baseline characteristics on physicians' decision to offer EVT to patients with M2 segment MCA occlusions revealed that the time from symptom onset to treatment, the baseline NIHSS, and the baseline ASPECTS were significant. This confirms that clinical and imaging baseline characteristics play an important role in clinical decision-making; the same variables that determine EVT eligibility in proximal-vessel occlusions ${ }^{6}$ are the variables that physicians rely on for decision-making in patients with M2 segment MCA occlusions.

Neither current nor ideal treatment decisions differed between academic and nonacademic centers in this survey. Physicians' treatment decisions, therefore, do not seem to be influenced by their institution's academic profile. Physicians who favor EVT under current local resources and under assumed ideal conditions worked in high EVT volume centers and performed significantly more EVT cases per year than those refraining from EVT. This reflects that experience with endovascular treatment makes physicians more comfortable offering EVT to their patients with M2 segment MCA occlusions. Interestingly, physicians who opt to use EVT in patients with M2 segment MCA occlusions were younger and had been in practice for fewer years than those who decided against using EVT. It is possible that these younger physicians were trained during the advent of stent retrievers and small distal aspiration catheters, techniques that make recanalization of M2 segment MCA occlusions safely achievable.

Significant differences were observed in reported EVT treatment decisions among the different geographic regions under current local resources but not under assumed ideal conditions (Fig 1B). Regions where physicians most preferred using EVT also showed the smallest gap between current and ideal treatment decisions, whereas regions with the lowest preference for using EVT showed the largest discrepancy between current and ideal decision rates. Respondents from Europe were less likely to offer EVT compared with the respondents from North America.

This could relate to access to EVT, differences in practice parameters and health care systems, differences in guidelines, and how practitioners implement these guidelines in their practice. Similarly, treatment decisions among different subspecialties under current local resources differed significantly but not under assumed ideal conditions (Fig 1C). These observations could point toward external factors (eg, insufficient access to treatment facilities, monetary and health policy-related limitations) as a possible cause for low EVT decision rates in some regions. For example, alteplase utilization remains $<1 \%$ in many developing countries. ${ }^{13}$ This may motivate the use of EVT in regions where patients or local jurisdictions are unable to afford alteplase. ${ }^{14}$ The gap between current and ideal practices in such regions may also reflect the limited resources of publicly funded hospitals or the patients' means in areas with mostly private hospitals. This could promote the use of manual aspiration at the clot interface as the primary and economically efficient EVT technique in local practices. ${ }^{13}$ In some other countries in Africa, Asia, and Europe, EVT is either not available or not offered around the clock. ${ }^{15}$ Such limitations with the subsequent impact on the access to EVT-capable facilities and devices are all important determinants of the decision for or against using EVT.

Although this study clearly shows that M2 segment MCA occlusions are considered an appropriate target for EVT across all countries and specialties, there was substantial variability in decision-making among physicians. This is not surprising because no randomized controlled trials demonstrated EVT efficacy in M2 segment MCA occlusions. To the best of our knowledge, there currently are no plans for such a trial. The main reason for this is the lack of clinical equipoise. Most physicians consider EVT the superior treatment for patients with all endovascular accessible occlusions, including the M2 segment; hence, only a few are willing to randomize patients with distal accessible occlusions.

Any future trial that seeks to enroll patients with M2 segment MCA occlusions, therefore, will need to take into account the lack of equipoise and the potential for bias in trial enrollment (patients with unfavorable risk profiles who are unlikely to benefit from EVT are more likely to get enrolled). Evidence from high-quality patient-level meta-analysis such as from the Highly Effective Reperfusion evaluated in Multiple Endovascular Stroke trials Collaboration ${ }^{7}$ and from cohort studies that enrolled patients with M2 segment MCA occlusions who have not been offered EVT such as the Identifying New Approaches to Optimize Thrombus Characterization for Predicting Early Recanalization and Reperfusion With IV Alteplase and Other Treatments Using Serial CT Angiography (INTERRSeCT) study, ${ }^{16}$ and numerous other nonrandomized studies ${ }^{4,8-11}$ may show the best available data for efficacy of EVT in such occlusions. In this situation, professional organizations and guideline committees may consider revising the endovascular treatment recommendations for M2 segment MCA occlusions despite the lack of Level-1A evidence. This will stimulate further improvement in the design of devices and aspiration catheters specifically tailored for distal vessels.

\section{Limitations}

Our study had several limitations. First, the survey respondents were contacted by using a purposive sampling strategy that relied on personal contacts with local principal investigators. The absence of a comprehensive international register of stroke physicians and neurointerventionalists means that it is not certain that the respondents were representative of their region or country. Although the overall survey completion rate was high, the number of responses for some countries and specialties was relatively low and, therefore, might not be fully representative of practices within that country or that specialty. Finally, physician decisions made in this survey were based on hypothetical scenarios and may not reflect decisions that may be taken in real life or for patients with different clinical or imaging parameters. Our survey does not account for some anatomic factors, for example, the diameter of the affected M2 artery, that may impact treatment decisions. Despite these limitations, our study provides valuable 
insights into current treatment approaches in patients with M2 segment MCA occlusions across different settings, regions, and specialties.

\section{CONCLUSIONS}

In the present study, EVT was considered the most likely selected treatment for patients with M2 segment MCA occlusions by practicing physicians.

\section{ACKNOWLEDGMENTS}

The authors thank the participants of this survey.

Disclosures: Johanna M. Ospel-RELATED: Grant: Julia Bangerer Rhyner Foundation, University of Basel Forschungsfonds, Freiwillige Akademische Gesellschaft Basel, Comments: Research scholarship. Gustavo SaposnikRELATED: Support for Travel to Meetings for the Study or Other Purposes: University of Calgary; UNRELATED: Consultancy: Roche, Servier, Celgene; Grants/Grants Pending: Roche, Servier*; Payment for Lectures Including Service on Speakers Bureaus: Celgene, Servier; Travel/Accommodations/ Meeting Expenses Unrelated to Activities Listed: Roche, Comments: Attendo to Ectrims 2019. Nima Kashani-UNRELATED: Employment: Calgary University. Andrew Demchuk-RELATED: Consulting fee or honorarium: Medtronic, Comments: CME events; UNRELATED: Patents (Planned, Pending or Issued): Circle NVI; Stock/stock options: Circle NVI. Michael D. Hill—RELATED: Grant: Stryker, Comments: Grant to the University of Calgary for the UNMASK-EVT project*; UNRELATED: Board Membership: CNSF board, CSC board, Comments: Not for profit boards in stroke and neurology; Consultancy: Merck, Comments: Paid consultancy for adjudicating clinical trial outcome events in clinical trials; Grants/Grants Pending: Multiple sources (public, industry), Comments: Multiple grants from both industry and public sources for clinical trials*; Patents (Planned, Pending or Issued): Patent in stroke care granted, Comments: Patent granted, no money paid; Stock/Stock Options: Calgary Scientific, Comments: Stock owner in CSI, imaging and computer rendering company. Mayank Goyal-RELATED: Grant: Stryker, Comments: Unrestricted research grant towards this study*; UNRELATED: Consultancy: Medtronic, Stryker, Mentice, Microvention; Patents (Planned, Pending or Issued): GE Healthcare, Comments: Licensing agreement for systems of acute stroke diagnosis. Bijoy K. Menon-Other Relationships: Holds a patent on systems of triage in acute stroke, holds stock in Circle Neurovascular Inc. *Money paid to institution.

\section{REFERENCES}

1. Hartmann A, Rundek T, Mast H, et al. Mortality and causes of death after first ischemic stroke: the Northern Manhattan Stroke Study. Neurology 2001;57:2000-05 CrossRef Medline

2. Koton S, Tanne D, Green MS, et al. Mortality and predictors of death 1 month and 3 years after first-ever ischemic stroke: data from the first National Acute Stroke Israeli Survey (NASIS 2004). Neuroepidemiology 2010;34:90-96 CrossRef Medline

3. Paolucci S, Antonucci G, Grasso MG, et al. Functional outcome of ischemic and hemorrhagic stroke patients after inpatient rehabilitation: a matched comparison. Stroke 2003;34:2861-65 CrossRef Medline

4. Rai AT, Domico JR, Buseman C, et al. A population-based incidence of M2 strokes indicates potential expansion of large vessel occlusions amenable to endovascular therapy. I Neurointerv Surg 2018;10:510-15 CrossRef Medline

5. Menon BK, Hill MD, Goyal M. Response by Menon to letter regarding article, "Analysis of workflow and time to treatment on thrombectomy outcome in the Endovascular Treatment for Small Core and Proximal Occlusion Ischemic Stroke (ESCAPE) randomized controlled trial." Circulation 2016;134:e406-07 CrossRef Medline

6. Powers WJ, Rabinstein AA, Ackerson T, et al. 2018 guidelines for the early management of patients with acute ischemic stroke: a guideline for healthcare professionals from the American Heart Association/American Stroke Association. Stroke 2018;49:e46-10 CrossRef Medline

7. Menon BK, Hill MD, Davalos A, et al. Efficacy of endovascular thrombectomy in patients with M2 segment middle cerebral artery occlusions: meta-analysis of data from the HERMES Collaboration. J Neurointerv Surg 2019;11:1065-69 CrossRef

8. Dorn F, Lockau H, Stetefeld H, et al. Mechanical thrombectomy of M2-occlusion. J Stroke Cerebrovasc Dis 2015;24:1465-70 CrossRef Medline

9. Grossberg JA, Rebello LC, Haussen DC, et al. Beyond large vessel occlusion strokes: distal occlusion thrombectomy. Stroke 2018;49:1662-68 CrossRef Medline

10. Coutinho JM, Liebeskind DS, Slater LA, et al. Mechanical thrombectomy for isolated M2 occlusions: a post hoc analysis of the STAR, SWIFT, and SWIFT PRIME studies. AJNR Am J Neuroradiol 2016;37:667-72 CrossRef Medline

11. Sheth SA, Yoo B, Saver JL, et al. M2 occlusions as targets for endovascular therapy: comprehensive analysis of diffusion/perfusion MRI, angiography, and clinical outcomes. J Neurointerv Surg 2015;7:478-83 CrossRef Medline

12. Saposnik G, Menon BK, Kashani N, et al. Factors associated with the decision-making on endovascular thrombectomy for the management of acute ischemic stroke. Stroke 2019;50:2441-47 CrossRef

13. Roa JA, Martins SCO, Mont'Alverne FJ. Stroke care and mechanical thrombectomy in developing countries. In: Samaniego E, Hasan D, eds. Acute Stroke Management in the Era of Thrombectomy. Cham: Springer-Verlag; 2019;203-20

14. Pandian JD, Sethi V, Dhillon R, et al. Is intravenous thrombolysis feasible in a developing country? Cerebrovasc Dis 2005;20:134-36 CrossRef Medline

15. Stevens E, Emmett E, Wang Y, et al. The Burden of Stroke in Europe. London : Stroke Alliance for Europe; 2017

16. Menon BK, Al-Ajlan FS, Najm M, et al. Association of clinical, imaging, and thrombus characteristics with recanalization of visible intracranial occlusion in patients with acute ischemic stroke. JAMA 2018;320:1017-26 CrossRef Medline 